\title{
MAGNETOLIPOSOMES BASED ON NICKEL/SILICA CORE/SHELL NANOPARTICLES: SYNTHESIS AND CHARACTERIZATION
}

Ana Rita O. Rodrigues, ${ }^{1}$ I. T. Gomes, ${ }^{1}$ Bernardo G. Almeida, ${ }^{1}$ J. P. Araújo, ${ }^{2}$ Elisabete M. S. Castanheira, ${ }^{1}$ and Paulo J. G. Coutinho, ${ }^{1, *}$

${ }^{I}$ Centro de Física (CFUM), Universidade do Minho, Campus de Gualtar, 4710-057 Braga, Portugal ${ }^{2}$ IFIMUP/IN - Instituto de Nanociência e Nanotecnologia, R. Campo Alegre, 4169-007 Porto, Portugal

\section{*Corresponding Author:}

Paulo J. G. Coutinho

Centro de Física, Universidade do Minho,

Campus de Gualtar,

4710-057 Braga, Portugal

Phone: +351 253 604321;

Fax: +351 253604061

pcoutinho@fisica.uminho.pt 


\begin{abstract}
In the present work, nickel magnetic nanoparticles with diameters lower than $100 \mathrm{~nm}$, with and without silica shell, were synthesized by microheterogeneous templating. The magnetic properties of the nanoparticles show a typical ferromagnetic behavior with a coercive field of 800e. Dry magnetoliposomes (DMLs) with diameter between 58nm and $76 \mathrm{~nm}$ were obtained from the synthesis of nanoparticles in the presence of a lipid or surfactant layer, and aqueous magnetoliposomes (AMLs) were obtained by encapsulation of the nanoparticles in liposomes. FRET (Förster resonance energy transfer) experiments were performed to study the non-specific interactions between aqueous magnetoliposomes and giant unilamellar vesicles (GUVs), as models of cell membranes. It was possible to detect membrane fusion between GUVs and AMLs containing both NBD-C 6 -HPC (donor) and the dye Nile Red (acceptor).
\end{abstract}

\title{
Highlights
}

1. Magnetic nickel nanoparticles were synthesized in microheterogeneous media.

2. The nanoparticles were covered with a silica shell to improve biocompatibility.

3. Aqueous and dry magnetoliposomes were prepared, the latter with diameter around $70 \mathrm{~nm}$.

4. Membrane fusion between magnetoliposomes and models of cell membranes was detected by FRET.

\section{Keywords}

Nanostructures; magnetic materials; interfaces; light scattering; photoluminescence spectroscopy; magnetic properties.

\section{Introduction}

Nanotechnology has produced significant advances in biomedicine, namely in diagnosis, therapy and bioengineering [1]. The potential of magnetic nanoparticles for biomedical applications has been recognized, as they offer major advantages due to their unique size and physicochemical properties [2].

Liposomes (nanosized vesicles made of amphiphilic phospholipid molecules in water) are biologically inert and weakly immunogenic, and have been described as ideal drug 
delivery systems [3-5]. This nanoencapsulation system can overcome many of the problems associated with other systems used for therapy, such as those involving solubility, pharmacokinetics, in vivo stability and toxicity [6,7]. Liposomes entrapping magnetic nanoparticles (magnetoliposomes) are of large importance in drug delivery, as they can be guided and localized to the therapeutic site of interest by external magnetic field gradients and used in cancer treatment by hyperthermia [8,9]. In diagnosis, magnetoliposomes have been proposed as T2 contrast agents (negative contrast enhancement) in MRI [10], while in therapy they have been used as a chemotherapy alternative through magnetic-controlled drug delivery and thermotherapy [11-13].

In biomedicine, nanoparticles with superparamagnetic behavior are preferred, as they exhibit a strong magnetization only when an external magnetic field is applied $[5,14,15]$. Iron and nickel nanoparticles are superparamagnetic when their size is smaller than a critical value, $20 \mathrm{~nm}$ for iron [16] and $30 \mathrm{~nm}$ for nickel [17]. Due to the wide applications of magnetoliposomes, much attention has been paid to the synthesis of different kinds of magnetic nanoparticles [18-20] and liposomes [14,21-23], as each potential application requires specific properties.

Nickel exhibits magnetic properties at room temperature and, therefore, is considered a metal of biological interest. However, particles of nickel have some issues such as potential toxicity, high reactivity and easy degradation due to the high surface/volume ratio. In order to overcome these problems and make them compatible for biological applications, nickel magnetic nanoparticles are typically protected by coatings, such as gold or silica, forming a core-shell structure [18,24,25]. Silica is a particular beneficial coating for nanoparticles, since it can easily be functionalized and it is resistant to degradation within a cellular environment, whilst still being biocompatible [26,27]. In fact, previous studies have shown that internalized silica-coated magnetic nanoparticles are biocompatible with stem cells [26,27].

The synthesis methods of magnetoliposomes and their constituents will determine their final shape, size distribution, surface chemistry and magnetic properties [15,28]. Techniques for magnetic nanoparticle synthesis have been developed to yield nearly monodisperse colloids, consisting of uniform nanoparticles both in size and shape. In these systems, the entire uniform physicochemical properties directly reflect the properties of single particles [29,30].

In this work, both aqueous and dry magnetoliposomes, based on nickel nanoparticles or nickel/silica core/shell nanoparticles were prepared by several soft templating methods 
and characterized. The interaction between the prepared magnetoliposomes and models of cell membranes (giant unilamellar vesicles, GUVs) was also evaluated using FRET (Förster Resonance Energy Transfer). These studies are important for future drug delivery applications using magnetoliposomes as drug carriers.

\section{Experimental}

All the solutions were prepared using spectroscopic grade solvents and ultrapure water (Milli-Q grade).

\subsection{Nickel nanoparticles preparation}

Ni nanoparticles (NPs) were prepared using a Nickel chloride (Merck) stock solution $(\sim 0.2 \mathrm{M})$. For $\mathrm{Ni}^{2+}$ reduction, hydrazine monohydrate $\left(\mathrm{N}_{2} \mathrm{H}_{4} \cdot \mathrm{H}_{2} \mathrm{O}\right) 64-65 \%$ and sodium hydroxide solution $(\mathrm{NaOH}, 50 \%$ in water), from Sigma-Aldrich, were used as received. Citric acid (Merck) was added in some assays in a molar ratio 1:0.75, as it prevents nanoparticles aggregation and favours monodispersity [28].

\subsubsection{Ni NPs in aqueous CTAB solution}

CTAB (cetyltrimethylammonium bromide) was obtained from Sigma-Aldrich. Ni NPs were synthesized in an aqueous solution of this cationic surfactant, according to a procedure adapted from [31]. An aqueous solution of CTAB (0.025 M), nickel chloride $(0.02 \mathrm{M})$ and trace acetone $(10 \mu \mathrm{L} / \mathrm{ml})$ was first prepared. Then, $1 \mathrm{M}$ of $\mathrm{N}_{2} \mathrm{H}_{4}$ and $10 \mathrm{M}$ $\mathrm{NaOH}$ solution were added in sequence. After 30 minutes at $60{ }^{\circ} \mathrm{C}$, Ni NPs were formed.

\subsubsection{Ni NPs coated with lipid or double-chain surfactant}

AOT (bis(2-ethylhexyl)sulfosuccinate) sodium salt from Sigma-Aldrich and DOPG (1,2-Dioleoyl-sn-glycero-3-[phospho-rac-(1-glycerol)]), from Avanti Polar Lipids, were used to control NPs size growth [32]. Ni NPs coated with a layer of AOT or DOPG molecules were synthesized by carrying out the reduction of nickel chloride in the presence of lipid/surfactant molecules. An experimental procedure previously described by Meledandri et al. [33] was followed. One fifth of the total DOPG amount (0.2 mM DOPG) was slowly added to a $1 \mathrm{mM}$ nickel chloride aqueous solution under magnetic 
stirring, followed by the addition of $0.9 \mathrm{M} \mathrm{N}_{2} \mathrm{H}_{4}$ and $13 \mathrm{M} \mathrm{NaOH}$ to precipitate the nickel. After 5 minutes, the remaining DOPG $(0.8 \mathrm{mM})$ was slowly added to the mixture. After about 20 minutes at $60^{\circ} \mathrm{C}$, Ni NPs covered by a DOPG layer were obtained [33].

Ni NPs covered with an AOT layer were synthesized by a similar procedure using a $56 \mathrm{mM}$ solution of AOT in methanol. After about 20 minutes, under vigorous magnetic stirring at $60^{\circ} \mathrm{C}$, NPs were formed. After cooling, Ni NPs were washed by magnetic decantation with methanol/acetone $(50 / 50 \mathrm{v} / \mathrm{v})$ solution and dispersed in water.

\subsubsection{NPs with silica shell}

The synthesized NPs were covered with a silica shell obtained by TEOS (tetraethyl orthosilicate, from Sigma Aldrich) hydrolysis. Different shell sizes were achieved by the addition of different amounts of TEOS into a solution of nanoparticles dispersed either in AOT/cyclohexane $(0.1 \mathrm{M})$ or in ethanol [34]. In the latter method, MDA (mercaptododecanoic acid) was added to the particles in a 1:1 ratio to promote TEOS binding to the nanoparticles.

\subsection{Preparation of magnetoliposomes}

Aqueous magnetoliposomes (AMLs) are formed when the magnetic nanoparticles are encapsulated in liposomes. Both dipalmitoyl phosphatidylcholine (DPPC) and egg yolk phosphatidylcholine (Egg-PC), from Sigma-Aldrich, were used for lipid vesicle formation. A $10 \mathrm{mM}$ DPPC or Egg-PC solution in ethanol was injected, under vigorous vortexing, to an aqueous solution of nanoparticles, above the melting transition temperature of the lipids (ethanolic injection method [33,36]). After encapsulation, the ferrofluid was washed with water and purified by magnetic decantation and centrifugation to remove all the non-encapsulated NPs.

Dry magnetoliposomes (DMLs) were synthesized by slowly adding a volume of DOPG or AOT solution, equivalent to that used in the synthesis of the NPs coated with lipid or surfactant, so that a second lipid/surfactant layer is formed above the previous layer. Excess of lipid/surfactant was removed by repeated cycles of magnetic decantation followed by washing with methanol/acetone (50/50 v/v) solution. 


\subsection{Preparation of Giant Unilamellar Vesicles (GUVs)}

Soybean lecithin ( $L$ - $\alpha$-Phosphatidylcholine), from Sigma-Aldrich, was used for GUVs preparation, using a procedure previously described [37,38]. A film of soybean lecithin was obtained by evaporation under an argon stream of a $1 \mathrm{mM}$ lipid solution. This film was incubated with $20 \mu \mathrm{L}$ of water at $45^{\circ} \mathrm{C}$ for 45 minutes. Then, $3 \mathrm{~mL}$ of $0.1 \mathrm{M}$ glucose solution was added, and the resulting mixture was again incubated at $37^{\circ} \mathrm{C}$.

\subsection{Spectroscopic measurements}

\subsubsection{General methods}

Absorption spectra were recorded in a Shimadzu UV-3101PC UV-vis-NIR spectrophotometer. Fluorescence measurements were performed using a Fluorolog 3 spectrofluorimeter, equipped with double monochromators in both excitation and emission and a temperature controlled cuvette holder. Fluorescence spectra were corrected for the instrumental response of the system.

\subsubsection{FRET measurements}

The interaction of magnetoliposomes with models of biological membranes (GUVs) was evaluated by Förster Resonance Energy Transfer (FRET). FRET efficiency, $\Phi_{\text {RET, }}$ defined as the proportion of donor molecules that have transferred their excess energy to acceptor molecules, was calculated through donor emission quenching, by taking the ratio of the donor integrated fluorescence intensities in the presence of acceptor and in the absence of acceptor [39]. The distance between donor and acceptor molecules was determined through the FRET efficiency (equation 1),

$$
r=R_{0} \cdot\left[\frac{1-\Phi_{\mathrm{RET}}}{\Phi_{\mathrm{RET}}}\right]^{1 / 6}
$$

where $R_{0}$ is the Förster radius (critical distance), that can be obtained by the spectral overlap between the donor emission and the acceptor absorption [39].

FRET assays were employed to confirm the formation of the second lipid bilayer in the dry magnetoliposomes (DMLs). For that purpose, the rhodamine B labeled lipid Rhodamine-DHPE (1,2-dipalmitoyl-sn-glycero-3-phosphoethanolamine- $N$-(lissamine rhodamine B sulfonyl) (ammonium salt) (from Avanti Polar Lipids, structure shown below - Figure 1) was included the first lipid layer, while the nitrobenzoxazole labeled 
lipid

NBD-C 6 -HPC

(1-palmitoyl-2-\{6-[(7-nitro-2-1,3-benzoxadiazol-4-

yl)amino]hexanoyl\}-sn-glycero-3-phosphocholine) (from Avanti Polar Lipids, Figure 1) was included in the second lipid layer.

For the study of the interaction of magnetoliposomes with GUVs, the former were labeled with both $\mathrm{NBD}-\mathrm{C}_{6}-\mathrm{HPC}$ and the hydrophobic probe Nile Red (from Fluka, structure in Figure 1).

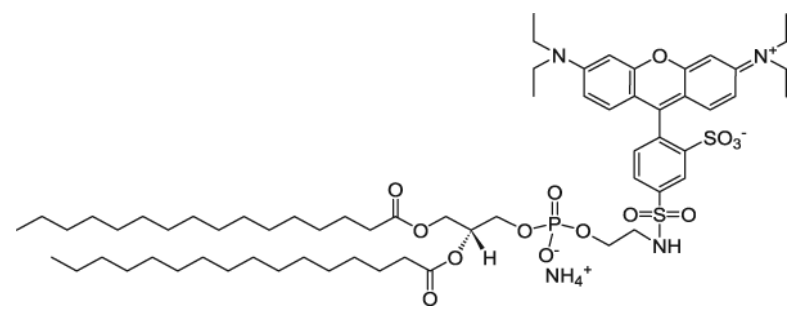

Rhodamine-DHPE
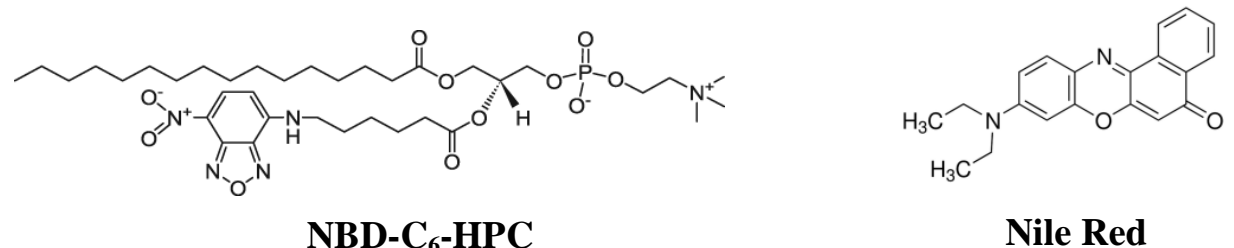

Nile Red

Figure 1 - Structures of the fluorescent labeled lipids and the dye Nile Red.

The fluorescence quantum yield, $\Phi_{s}$, of the energy donor (in both cases, the dye NBD) in magnetoliposomes was determined by the standard method (equation 2) [40,41],

$$
\Phi_{s}=\frac{A_{r} F_{S} n_{S}^{2}}{A_{S} F_{r} n_{r}^{2}} \Phi_{r}
$$

where $A$ is the absorbance at the excitation wavelength, $F$ the integrated emission area and $n$ the refraction index of the solvents used. Subscripts refer to the reference (r) or sample (s). The absorbance at the excitation wavelength was always lower than 0.1 to

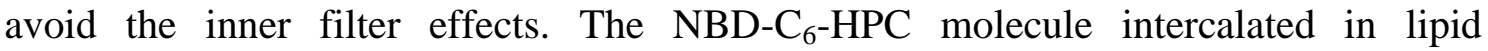
membranes was used as reference, $\Phi_{\mathrm{r}}=0.32$ at $25^{\circ} \mathrm{C}$, as reported by Invitrogen [42].

\subsection{Structural and magnetic characterization}

Scanning electron microscopy (SEM) images of nickel nanoparticles and dry magnetoliposomes were recorded using a Scanning Electron Microscope FEI - Nova 200 NanoSEM. The processing of SEM images was performed using ImageJ software. 
It consisted in enhancing local contrast followed by automatic local thresholding and particle analysis. The area of each particle allowed an estimation of the particle diameter. The resulting histograms were fitted to Gaussian distributions.

Magnetic hysteresis cycles of nickel NPs were measured at room temperature in a Superconducting Quantum Interference Device (SQUID) magnetometer (Quantum Design MPMS5XL), with applied magnetic fields up to $5.5 \mathrm{~T}$.

NPs mean diameter and size distribution (polydispersity index) were measured using a Dynamic Light Scattering (DLS) equipment (NANO ZS Malvern Zetasizer) at $25^{\circ} \mathrm{C}$, using a He-Ne laser of $\lambda=632.8 \mathrm{~nm}$ and a detector angle of $173^{\circ}$. Five independent measurements were performed for each sample.

The experimentally obtained intensity autocorrelation function, $G^{(2)}(\tau)$, has the form $[43,44]$

$$
G^{(2)}(\tau)=A\left[1+B\left|g^{(1)}(\tau)\right|^{2}\right]
$$

where $A$ is the baseline, $B$ is a spatial coherence factor, $\tau$ is the delay time, and $g^{(1)}(\tau)$ is the first-order normalized electric field time correlation function.

For a polydisperse system, $g^{(1)}(\tau)$ decays as a weighted sum of single exponentials. These weights are modeled by Gaussian distributions, such that

$$
g^{(1)}(\tau)=\int_{0}^{\infty} \sum_{i} \frac{a_{i}}{\sigma_{i} \sqrt{2 \pi}} \exp \left(-\left[\frac{\left(\mathrm{s}-\mathrm{s}_{i}\right)}{\sqrt{2} \sigma_{i}}\right]^{2}\right) \exp (-\tau / s) \mathrm{ds}
$$

where $a_{i}$ is the weight of each Gaussian population.

The decay lifetime, $s$, depends on the translational diffusion coefficient, $\mathrm{D}_{\mathrm{z}}$, which can be related to the particle hydrodynamic diameter through the Stokes-Einstein equation. A "size distribution" can thus be obtained, which is represented by $G_{I}(d)$, as each particle population is weighted by the intensity of scattered light it originates. In order to obtain the real size distribution of the sample, in which each particle population is weighted by its number fraction, $G_{n}(d)$, the variation of light scattering intensity with particle size (and shape) must be introduced [45],

$$
G_{I}(d)=G_{n}(d) I_{s}(d)
$$

where $I_{S}(d)$ is the scattered intensity per particle.

For spherical particles, and in the case of Rayleigh regime (valid for $x=\pi d / \lambda \ll 1$ ), this factor scales with $d^{6}$. For situations where the refractive index of the particles and the medium are similar, the Rayleigh-Gans-Debye (RGD) theory is valid and the 
scattering light intensity is proportional to the square of the particle volume and to a form factor, $\mathrm{P}(\mathrm{R})=\mathrm{f}(\mathrm{R})^{2}$ [46]. For other situations, the value of $I_{s}(d)$ must be calculated using appropriate theories, such as Mie theory (spherical particles) or Aden-Kerker theory (spherical coated particles).

\section{Results and discussion}

\subsection{Characterization of Ni nanoparticles}

\subsubsection{Absorption spectra}

Figure 2 shows the absorption spectrum of nickel nanoparticles obtained from the synthesis in CTAB microemulsions, without (A) and with silica shell (B). Considering the reported cytotoxicity of Ni NPs for several human cell lines $[47,48]$, the presence of the silica shell could be important for biomedical applications of these magnetic nickel nanoparticles. As referred, the biocompatibility of silica nanoparticles and magnetic nanoparticles with a silica-shell was previously demonstrated [26,27].

The formation of Ni metal nanoparticles is confirmed by the absorption in the whole spectral range and plasmon absorption band at $288 \mathrm{~nm}$ (Figure 2). This plasmon absorption band is very sensitive to the local dielectric environment and results from a resonant coherent oscillation of the free electrons at the surface of a spherical NP (LSPR - localized surface plasmon resonance) that is induced by the electromagnetic field of incident light [49].

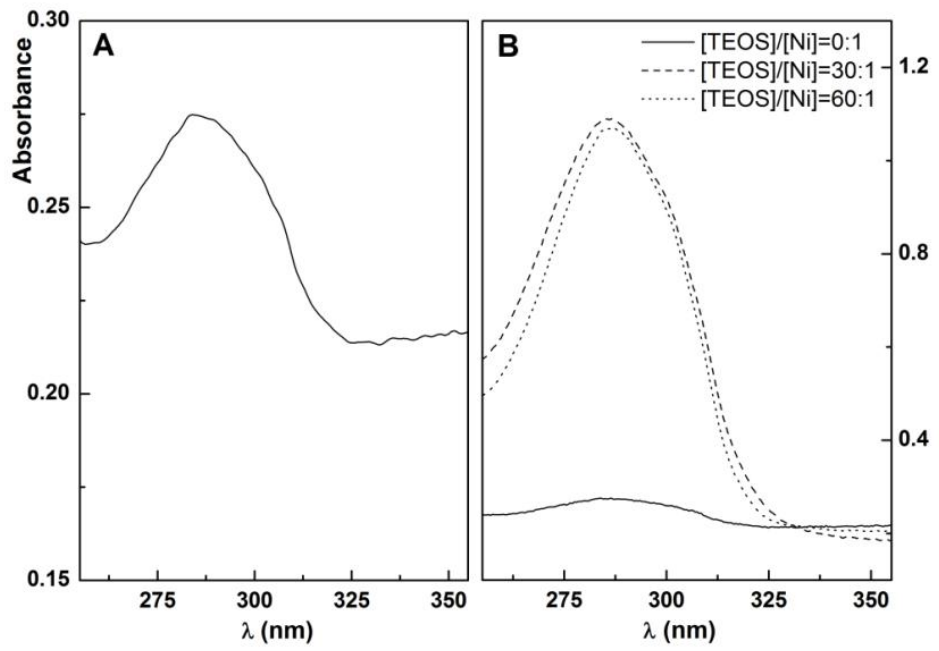

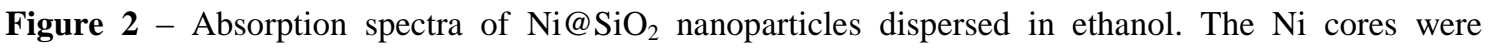
synthesized in aqueous CTAB solution. A: without silica shell; B: with silica shell added to Ni NPs dispersed in AOT/cyclohexane using different [TEOS]/[Ni] ratios. 
Core-shell nickel nanoparticles with different silica shell sizes were formed by the addition of TEOS at several concentrations. A strong SPR enhancement is observed after coating (Figure 2B), indicating a change in the dielectric constant of the medium surrounding the NPs, confirming formation of the silica shell (Figure 2B). However, no further increase in absorption intensity was observed for [TEOS]/[Ni] molar ratio above 30:1. This can be attributed to the method used for the formation of the silica shell. As TEOS is added to the Ni NPs dispersed in a AOT/cyclohexane solution, the sol-gel process occur within AOT reverse micelles. This can limit the shell growth. For gold nanoparticles, very small changes are observed in the plasmon band [50]. On the other hand, a band near $270 \mathrm{~nm}$ was reported for $\mathrm{SiO}_{2}$ nanoparticles obtained from sol-gel processing [51]. Thus, the huge absorbance increase upon coating of Ni NPs with $\mathrm{SiO}_{2}$ should originate from absorption and/or scattering of the silica layer.

\subsubsection{DLS measurements}

DLS measurements revealed that particles size and size distribution are influenced by the synthesis method. Hydrodynamic diameters of Ni NPs with different [silica]/[Ni] ratios and without silica shell are shown in Table 1.

The scattered light per particle, $I_{s}(d)$, needed to obtain the real size distributions from DLS data, was calculated using the equations proposed by Aden-Kerker [52] with a Fortran implementation developed by Quirantes et al. [53]. Figure 3 plots $\mathrm{s}_{11}$, an element of the scattering matrix (proportional to $\mathrm{I}_{\mathrm{s}} / \mathrm{I}_{0}[46]$ ), as function of particle diameter for unpolarized light of $632.8 \mathrm{~nm}$ at $173^{\circ}$ scattering angle and a bulk refractive index of 1.36042 (ethanol at $25^{\circ} \mathrm{C}$ ). For Ni NPs, a refractive index of $\mathrm{m}(\mathrm{Ni})=1.97006+$ $3.72121 i$ [54] was used. In the case of $\mathrm{Ni} @ \mathrm{SiO} 2$ core/shell NPs, a value of 1.45702 [55] was used for the refractive index of silica, either considering a constant Ni core with a diameter of $100 \mathrm{~nm}$, or a constant $\mathrm{SiO}_{2}$ shell of $50 \mathrm{~nm}$ thickness. For vesicles, a bilayer with $5 \mathrm{~nm}$ thickness was used with a refractive index of 1.435 [56].

It is observed that Rayleigh regime is only valid for Ni NPs up to $20 \mathrm{~nm}$ diameter. The RGD approximation is better, but the oscillations do not occur at the same particle sizes and the minima are much more pronounced. In the case of vesicles, the RGD approximation is quite good. 


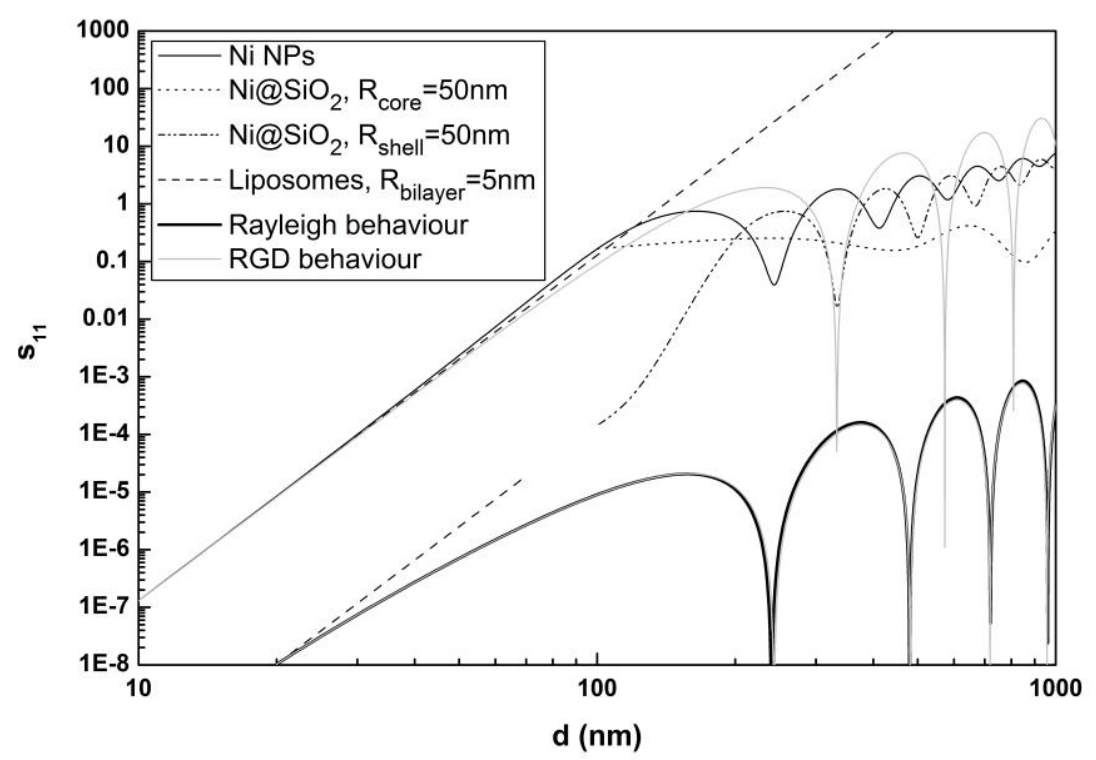

Figure 3- $\mathrm{S}_{11}$ scattering matrix element for $\mathrm{Ni} \mathrm{NPs}, \mathrm{Ni} @ \mathrm{SiO}_{2} \mathrm{NPs}$ and vesicles.

The number weighted size distributions could then be obtained by fitting a Gaussian distribution to the calculated $G_{n}(d)$ from equation (5). In the case of $\mathrm{Ni} @ \mathrm{SiO}_{2}$, a Gaussian shell size distribution was considered superimposed on the distribution obtained for bare Ni NPs and a sum was calculated for different particles having the same total diameter. The results of this procedure are shown in Table 1 and the recovered mean size are approximately $4 \mathrm{~nm}$ less than those obtained from intensity weighted size distributions, $G_{I}(d)$. The eventual effect of the $\sim 2 \mathrm{~nm}$ AOT or DOPG overcoat was not considered.

Comparing the hydrodynamic diameter of Ni NPs with and without silica shell, it is possible to confirm the formation of the shell based on size increase with the $[\mathrm{TEOS}] /[\mathrm{Ni}]$ ratio. Above $[\mathrm{TEOS}]:[\mathrm{Ni}]=30: 1$, the increase of the shell size is smaller, which can be caused by the limited space of the water pools inside AOT reverse micelles (as already referred), that in fact control the particles size. Core/shell nanoparticles are generally more polydisperse, as inferred from a larger size distribution.

The smallest particles, with hydrodynamic diameters in the order of $80 \mathrm{~nm}$ and narrow size distribution, were obtained from the synthesis of NPs (without silica shell) covered with a layer of the phospholipid DOPG. This shows that the lipid layer contributes to avoid particle aggregation and to reduce size and polydispersity. However, some degree 
of aggregation is expected in these systems, as the lipid/surfactant layer is supposed to have the hydrophobic chains turned to the outer phase.

Table 1. Hydrodynamic diameter (obtained by DLS) of Ni nanoparticles with and without silica shell, prepared by several synthesis methods.

\begin{tabular}{|c|c|c|c|c|}
\hline \multirow{3}{*}{ Ni NPs synthesis method } & \multirow{3}{*}[\mathrm{TEOS}]{$:[\mathrm{Ni}]$} & \multicolumn{3}{|c|}{ Hydrodynamic diameter (nm) } \\
\hline & & \multirow{2}{*}{$\begin{array}{l}\text { Intensity } \\
\text { Distribution }\end{array}$} & \multicolumn{2}{|c|}{ Number Distribution } \\
\hline & & & Core & $\begin{array}{c}\text { Shell } \\
\text { thickness }\end{array}$ \\
\hline \multirow{4}{*}{$\begin{array}{l}\text { Aqueous CTAB solution } \\
\text { TEOS added in AOT/cyclohexane }\end{array}$} & $0: 1$ & $88 \pm 7$ & $84 \pm 7$ & --- \\
\hline & $10: 1$ & $157 \pm 16$ & $84 \pm 7$ & $34 \pm 7$ \\
\hline & $30: 1$ & $175 \pm 24$ & $84 \pm 7$ & $42 \pm 12$ \\
\hline & $60: 1$ & $185 \pm 21$ & $84 \pm 7$ & $48 \pm 10$ \\
\hline Covered with one AOT layer & $0: 1$ & $100 \pm 9$ & $95 \pm 13$ & --- \\
\hline Covered with one DOPG layer & $0: 1$ & $79 \pm 6$ & $76 \pm 6$ & --- \\
\hline
\end{tabular}

\subsubsection{SEM microscopy}

Scanning Electron Microscopy (SEM) allow the direct observation of 2D projections of NPs structure, surface morphology and size. SEM images generally revealed nanoparticles with radius lower than $100 \mathrm{~nm}$ for almost all the synthesis methods employed. The NPs synthesized in CTAB aqueous solution revealed diameters between 63.4 and $104 \mathrm{~nm}$ (Figure 4), with a size distribution of $66 \pm 24 \mathrm{~nm}$ obtained from the histogram of image B. This result is slightly lower than the size distribution obtained from DLS measurements, indicating particle dimerization in aqueous media. Phase contrast SEM images (Figure 4C versus Figure 4D) confirmed that the particles obtained are metal nanoparticles. 

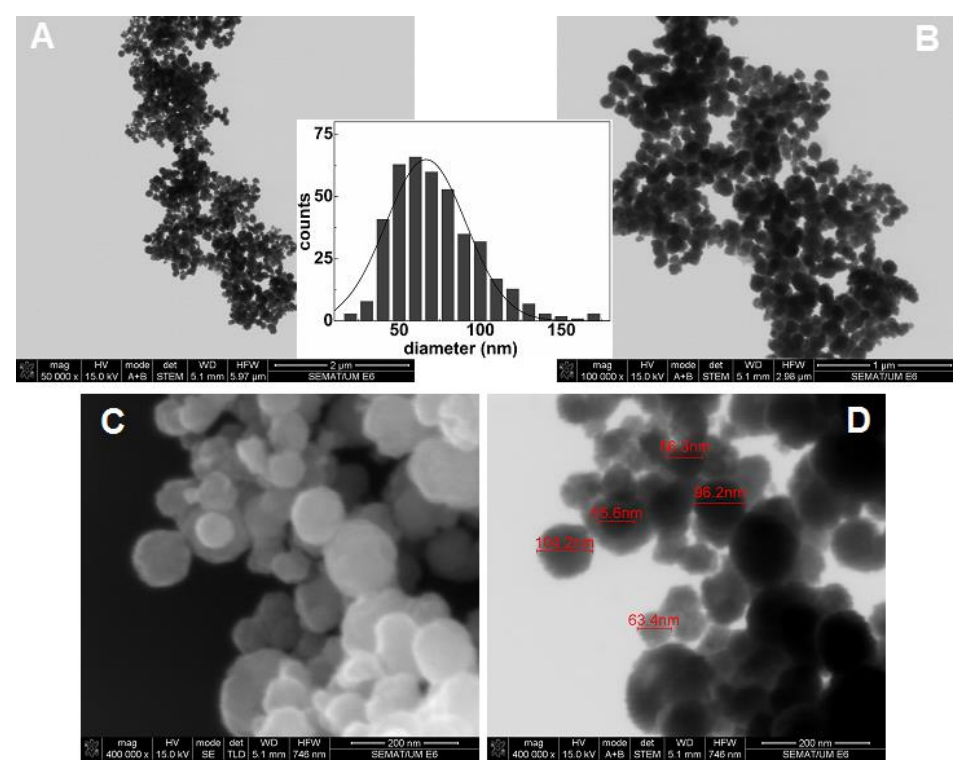

Figure 4 - SEM images of nickel nanoparticles synthesized in CTAB aqueous solution with citric acid (1:0.75), at different amplifications. Inset: Particles size histogram of image $\mathbf{B}$ and fitting to a Gaussian distribution.

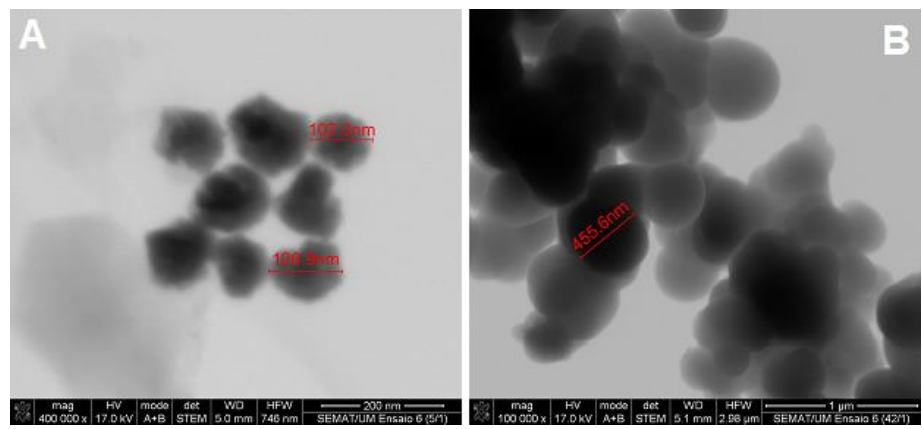

Figure 5 - SEM images of nickel nanoparticles coated with silica shell. A: [TEOS]/[Ni]=5:1 (TEOS added in AOT/cyclohexane solution); B: [TEOS]/[Ni]=42:1 (TEOS added in ethanol solution with MDA).

The increase in size of the shell is proportional to TEOS concentration and is dependent on the synthesis process (Figure 5). As referred, two processes were used for the coating with silica shell. In the first, TEOS was added in an AOT solution in cyclohexane, and the shell growth is controlled by the size of water pools of AOT reverse micelles. In the second, shell growth is not limited, as TEOS is added in ethanol together with a binding agent (MDA). A disadvantage of the first method is the low NPs concentration obtained (Figure 5A). As expected, the second method yields particles with a much larger silica shell, attaining more than 400nm diameter for high [TEOS]/[Ni] ratio (Figure 5B). 


\subsubsection{Magnetic Properties}

The magnetic properties of the nanoparticles synthesized in CTAB aqueous solution were characterized by measuring their corresponding magnetic hysteresis loop, which shows the relationship between the induced magnetic moment and the applied magnetic field $(\mathrm{H})$. Figure 6 shows the hysteresis cycle measured on the prepared nickel nanoparticles. Typical ferromagnetic properties were observed, reaching the saturation at about $1 \mathrm{kOe}(0.1 \mathrm{~T})$. The nickel nanoparticles exhibit some slight hysteresis (Figure 6B), indicating an oxidation of the particles, with the formation of a $\mathrm{NiO}$ (antiferromagnetic) surface layer around the metallic particles. In fact, the obtained hysteresis loop (Figure 6A) is identical to the one previously reported for Ni NPs with a $\mathrm{NiO}$ layer [57]. The coercive field of the Ni NPs is 80 Oe (Figure 6B - point c) and is lower than the value reported, at room temperature, for NPs with NiO layer prepared by similar methods, which indicates a smaller amount of nickel oxidation on our prepared particles.

In order to estimate the thickness of the $\mathrm{NiO}$ layer from the magnetic hysteresis cycles, the particles were considered to have a well ordered Ni core covered by a non-magnetic $\mathrm{NiO}$ shell (with thickness $\delta$ ) that acted as a magnetic "dead layer" [58]. In this respect, the measured saturation magnetization $\left(\mathrm{M}_{\mathrm{s}}\right)$ of the particles is proportional to the volume fraction of the core, which carries the spontaneous magnetization. If the shell thickness $\delta$ is small, then the saturation magnetization can be determined, to first order, by [58]

$$
M_{s}=M_{s 0}\left(1-\frac{6 \delta}{D}\right)
$$

where $\mathrm{D}$ is the particle diameter and $\mathrm{M}_{s 0}$ is the saturation magnetization for a bulk $\mathrm{Ni}$ sample $\left(\mathrm{M}_{s 0}=55 \mathrm{emu} / \mathrm{g}\right)$. Here, for particles with diameter of $84 \mathrm{~nm}$ and with $\mathrm{M}_{s}=51.2$ $\mathrm{emu} / \mathrm{g}$, the obtained $\mathrm{NiO}$ layer thickness is $\delta=1.1 \mathrm{~nm}$, which corresponds to about 3 unit cells. This shows that the synthesis of Ni particles using the cationic surfactant CTAB allows attaining an improvement of the NPs magnetic properties, relative to previous methods employing Triton X-100 as surfactant [57]. 

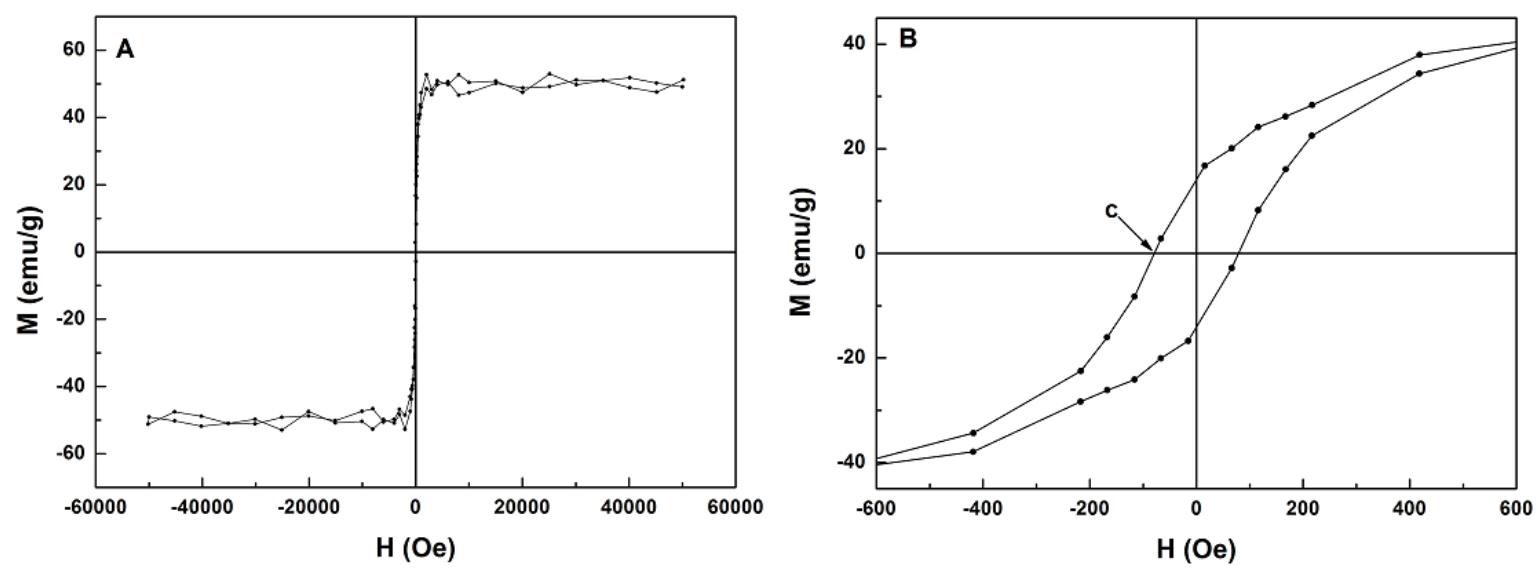

Figure 6 - A: Magnetization hysteresis cycle of the nickel nanoparticles at room temperature.

B: Enlargement of the hysteresis loop of Figure 6A, in the low field region.

\subsection{Characterization of magnetoliposomes}

\subsubsection{FRET assays in DMLs}

As described in the Experimental Section, two types of magnetoliposomes were synthesized, aqueous magnetoliposomes (AMLs) and dry magnetoliposomes (DMLs). In DMLs, clusters of magnetic nanoparticles were covered by the double chain surfactant AOT or the anionic phospholipid DOPG. The coating by a double lipid (or surfactant) layer was confirmed by FRET assays. The NDB labeled lipid NBD-C 6 -HPC, included in the second lipid layer of the DMLs, acts as energy donor, while the labeled lipid Rhodamine B-DHPE was included in the first lipid/surfactant layer, acting as energy acceptor.

Figure 7 presents evidence for FRET occurrence between NBD and Rhodamine (Rh). Inset illustrates the spectral overlap between the emission of the donor (NBD) and the absorption of the acceptor (Rhodamine B), indispensable condition for FRET to occur. Fluorescence spectra of DMLs containing only donor or acceptor and DMLs containing both labeled lipids were measured, exciting only the donor NBD $\left(\lambda_{\text {exc }}=465 \mathrm{~nm}\right)$, at the same dilution factor. As expected, characteristic NBD emission $\left(\lambda_{\mathrm{em}}=520 \mathrm{~nm}\right)$ is detected for DMLs labeled only with NBD-C 6 -HPC, while negligible fluorescence is observed for the DMLs containing only Rhodamine-DHPE. It is possible to verify that for DMLs with both donor and acceptor molecules, the emission in the NBD-C 6 -HPC region notably decreases, with a strong rise in the Rhodamine $\mathrm{B}$ region, proving the formation of the second lipid layer in DMLs. 


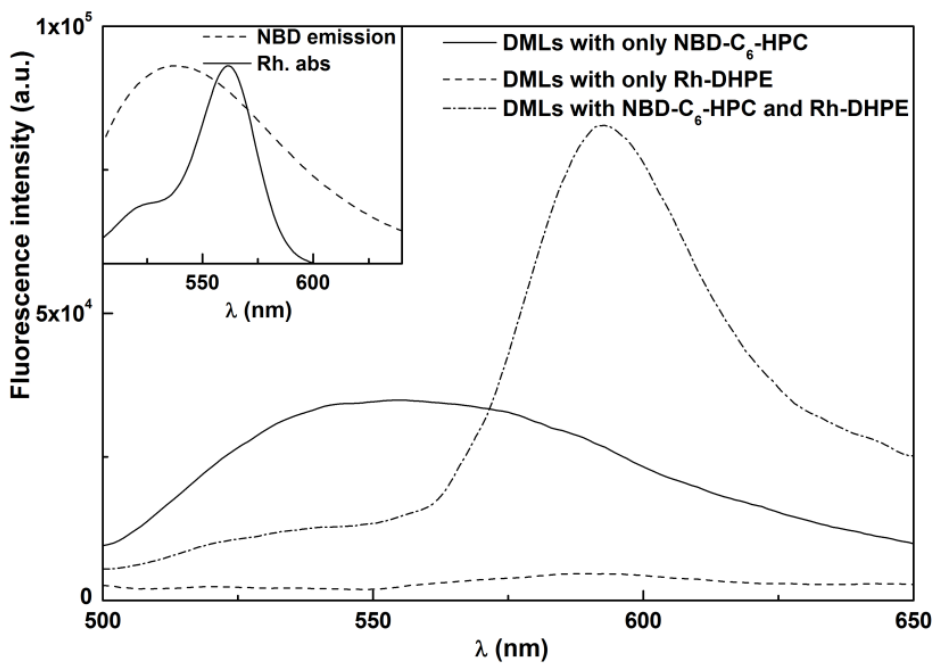

Figure 7 - Fluorescence spectra $\left(\lambda_{\mathrm{exc}}=465 \mathrm{~nm}\right.$, no Rhodamine excitation) of DMLs covered with AOT labeled with only NBD-C 6 -HPC $\left(1 \times 10^{-2} \mu \mathrm{M}\right)$; DMLs labeled with only Rhodamine B-DHPE $\left(1 \times 10^{-2} \mu \mathrm{M}\right)$ and DMLs labeled with both NBD-C 6 -HPC $\left(1 \times 10^{-2} \mu \mathrm{M}\right)$ and Rhodamine B-DHPE $\left(1 \times 10^{-2} \mu \mathrm{M}\right)$. Inset: Spectral overlap (spectra are normalized) between the fluorescence emission of the donor (NBD-C ${ }_{6}-\mathrm{HPC}$ ) and the absorption of the acceptor (Rhodamine B-DHPE).

Using the standard method, the fluorescence quantum yield of the donor in magnetoliposomes (in the absence of acceptor) was determined as $\Phi_{D}=0.185$. FRET measurements revealed an energy transfer efficiency of $31 \%$, with a corresponding donor-acceptor distance of $7.6 \mathrm{~nm}$. Cell membrane has a typical thickness of 7 to $9 \mathrm{~nm}$ [59]. Therefore, these results clearly indicate that the labeled lipids Rhodamine BDHPE and NBD- $\mathrm{C}_{6}-\mathrm{HPC}$ are placed in the first and second lipid layer, respectively, in the structures formed. This confirms the structure of lipid bilayer around nickel nanoparticles and the synthesis of DMLs.

\subsubsection{SEM microscopy and DLS measurements}

Dry magnetoliposomes (DMLs) can be observed by SEM microscopy, as their structure does not present an inner water pool. On the contrary, the structure of aqueous magnetoliposomes (AMLs) is destroyed by the vacuum system used in SEM.

The sizes of AMLs, prepared by using either DPPC or Egg-PC phospholipids, were determined by DLS (Table 2). The size of liposomes (without Ni NPs) is also shown, for comparison. 
Table 2. Hydrodynamic diameter (obtained by DLS) of aqueous magnetoliposomes incorporating Ni NPs with and without silica shell.

\begin{tabular}{|c|c|c|c|}
\hline \multirow[b]{2}{*}{ Phospholipid } & \multirow[b]{2}{*}[\mathrm{TEOS}]{$:[\mathrm{Ni}]$} & \multicolumn{2}{|c|}{ Hydrodynamic diameter (nm) } \\
\hline & & $\begin{array}{c}\text { Intensity } \\
\text { distribution }\end{array}$ & $\begin{array}{c}\text { Number } \\
\text { distribution } \\
\text { (fixed shell of } 5 \mathrm{~nm} \\
\text { thickness) }\end{array}$ \\
\hline \multirow{4}{*}{ Egg-PC } & without NPs & $92 \pm 10$ & $90 \pm 7$ \\
\hline & $0: 1$ & $103 \pm 20$ & --- \\
\hline & $10: 1$ & $126 \pm 33$ & --- \\
\hline & $20: 1$ & $135 \pm 34$ & --- \\
\hline \multirow{3}{*}{ DPPC } & without NPs & $115 \pm 12$ & $113 \pm 9$ \\
\hline & $0: 1$ & $146 \pm 34$ & --- \\
\hline & $20: 1$ & $175 \pm 23$ & --- \\
\hline
\end{tabular}

As previously reported, the size of Egg-PC liposomes is usually smaller than the one for DPPC liposomes prepared by the same technique (ethanolic injection) [60]. The results on Table 2 show that the presence of Ni nanoparticles contributes to a diameter increase relative to liposomes without NPs, this effect being more significant in DPPC magnetoliposomes.

The entrapment of core/shell Ni/silica nanoparticles in liposomes contribute definitely to avoid particle aggregation, as the measured mean diameters are significantly lower for AMLs than for the corresponding nanoparticles ( $v d$. Table 1). Comparing both lipid systems, the Egg-PC AMLs are more promising for applications in drug transport and delivery, considering their size and size distribution.

SEM micrographs of DMLs of Ni nanoparticles covered by a double layer of the anionic surfactant AOT or of the phospholipid DOPG are presented in Figures 8 and 9, respectively. Figure 8B shows AOT DMLs with size varying between 58 and $76 \mathrm{~nm}$, with a relatively low polydispersity. From the fit to a Gaussian distribution of the particles size histogram obtained from the image processing of Figure 8A (inset of Figure 8), a particle diameter of $67 \pm 26 \mathrm{~nm}$ was calculated. DLS measurements allowed to determine a hydrodynamic diameter of $115 \pm 26 \mathrm{~nm}$, pointing to extended aggregation in aqueous media. 


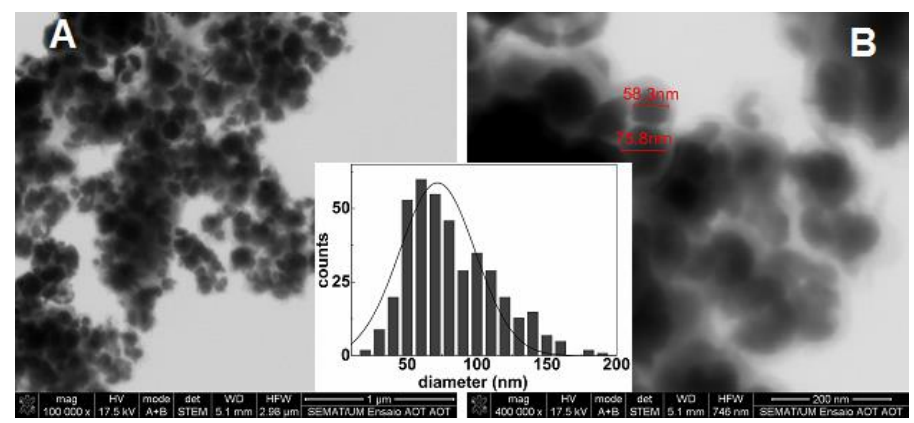

Figure 8 - SEM images of dry magnetoliposomes of nickel nanoparticles (without silica shell) covered by an AOT surfactant double layer. Inset: Particles size histogram of image A and fitting to a Gaussian distribution.

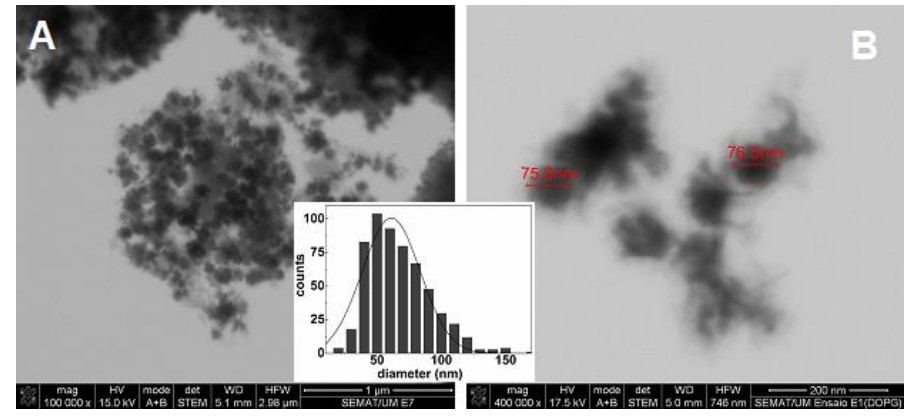

Figure 9 - SEM images of dry magnetoliposomes of nickel nanoparticles covered by a DOPG lipid bilayer. Inset: Particles size histogram of image $\mathbf{A}$ and fitting to a Gaussian distribution.

SEM results revealed that the DOPG DMLs are approximately monodisperse (Figure 9B), with size around $76 \mathrm{~nm}$. The fit to a Gaussian distribution of the particles size histogram obtained from SEM images (Figure 9A) allowed determining a diameter of $65 \pm 28 \mathrm{~nm}$. DLS measurements revealed one population with a hydrodynamic diameter of $98 \pm 16 \mathrm{~nm}$, pointing again to some dimerization in aqueous media.

These results are promising for future application of DMLs as drug transport/delivery systems, specially the DOPG-based system, as its size is below $100 \mathrm{~nm}$ with a narrow size distribution.

\subsection{Interaction of magnetoliposomes with giant unilamellar vesicles (GUVs)}

Non-specific interactions of the aqueous magnetoliposomes (AMLs) with giant unilamellar vesicles (GUVs), models of cell membranes, were also evaluated by FRET. For that purpose, the labeled lipid NBD-C 6 -HPC was included in Egg-PC AMLs, acting 
as energy donor, while the hydrophobic probe Nile Red, also incorporated in AMLs, acts as acceptor.

Nile Red is a well-known solvatochromic probe, which in polar media exhibits a red shift in the emission maximum, together with fluorescence quenching. Owing to its capability to establish H-bonds with protic solvents, Nile Red fluorescence in water is very weak and red shifted $\left(\lambda_{\max } \sim 660 \mathrm{~nm}\right)$ [61]. Nile Red has been used as a lipid probe, due to its hydrophobic nature [62-65].

The significant overlap between NBD- $\mathrm{C}_{6}$-HPC emission band and Nile Red absorption spectrum (Figure 10 - inset) indicates that FRET process between these two fluorescent molecules is expected to be efficient, if the donor-acceptor distance is below $100 \AA$ [39]. In fact, when both fluorophores are incorporated in magnetoliposomes at appropriate surface densities, efficient energy transfer is observed, exciting only the donor (NBD) (Figure 10). Two fluorescence bands are observed, the first $\left(\lambda_{\max }=535\right.$ $\mathrm{nm})$ corresponding to NBD-C 6 -HPC emission and the second one to Nile Red, with maximum at $630 \mathrm{~nm}$. This second band arises from the energy transfer of excited NBD molecules to Nile Red. When the magnetoliposomes interact with GUVs, if fusion occurs, a larger membrane is formed [66]. This leads to an increase in the donoracceptor distance and a corresponding decrease in the energy transfer efficiency from the NBD moieties, as is experimentally observed.

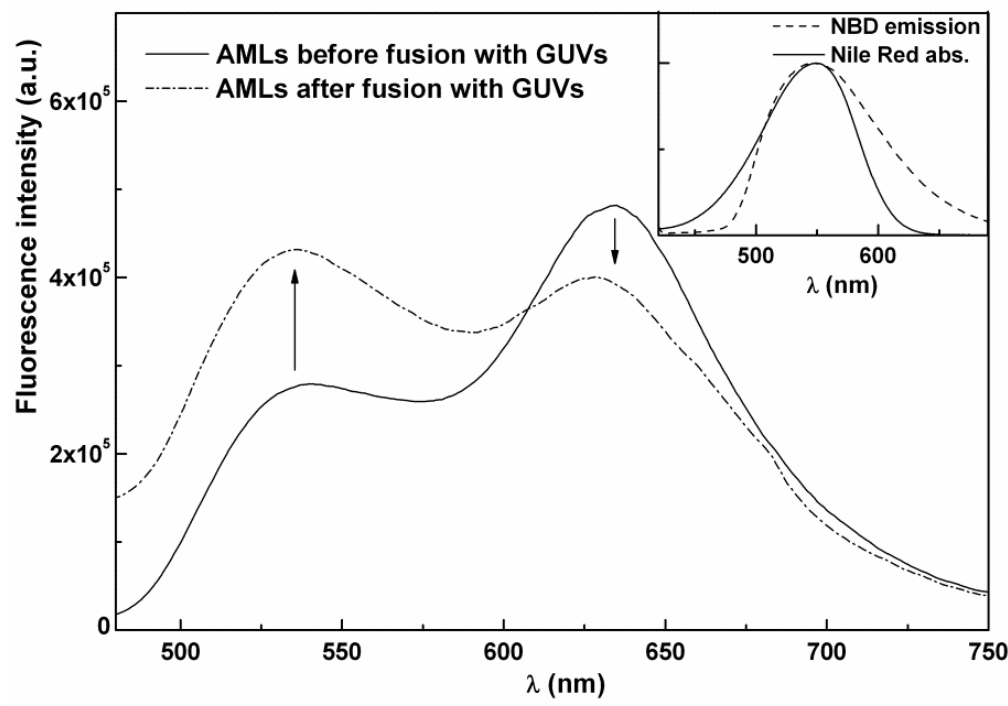

Figure 10 - Fluorescence spectra $\left(\lambda_{\text {exc }}=400 \mathrm{~nm}\right)$ of AMLs of Egg-PC and Ni/silica core/shell NPs containing both NBD-C $\mathrm{C}_{6}$ HPC $\left(10^{-6} \mathrm{M}\right)$ and Nile Red $\left(2 \times 10^{-6} \mathrm{M}\right)$, before and after interaction with GUVs. Inset: Spectral overlap (spectra are normalized) between the fluorescence emission of the donor (NBD$\mathrm{C}_{6}$-HPC) and the absorption of the acceptor (Nile Red). 
These experimental results are consistent with membrane fusion between the AMLs and GUVs. This process of membrane fusion is illustrated in Figure 11.

Thus, a proof-of-concept is presented in this work, allowing to conclude that both aqueous and dry magnetoliposomes may be used as drug transport and delivery systems, as they can be guided with a magnetic field and can release the encapsulated drugs by fusion with the cell membrane.

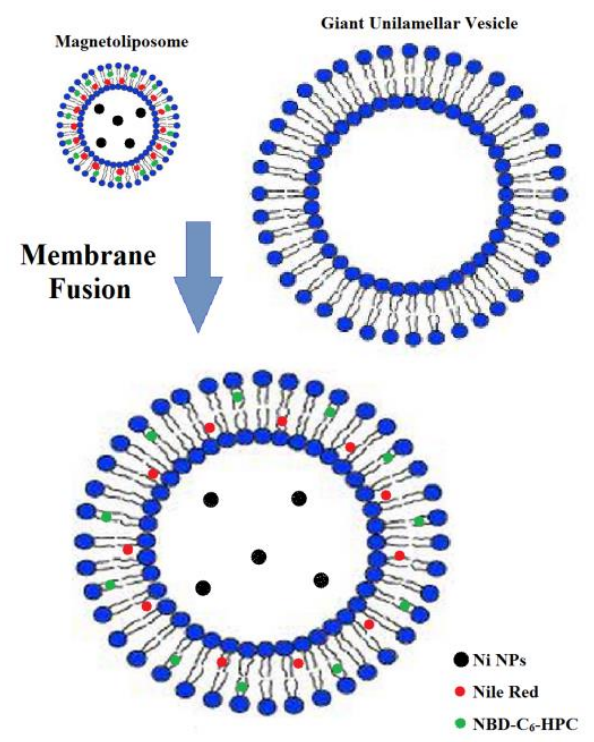

Figure 11 - Schematic illustration of the fusion between the GUVs and magnetoliposomes labeled with both NBD-C 6 -HPC and Nile Red.

\section{Conclusions}

In this work, magnetic nickel nanoparticles were successfully synthesized using microheterogeneous templating media.

SQUID measurements of Ni NPs synthesized in CTAB aqueous solution allowed to determine a coercive field of 80 Oe.

Nickel nanoparticles were successfully encapsulated into liposomes, forming aqueous (AMLs) or dry magnetoliposomes (DMLs). DMLs with AOT surfactant or DOPG double chain present low polydispersity and mean diameter lower than $100 \mathrm{~nm}$, essential for in vivo applications. FRET measurements point to membrane fusion between the magnetoliposomes and models of cell membranes (GUVs).

These results may be important for future drug delivery applications of antitumor drugs using magnetoliposomes for encapsulation and transport of antitumor drugs and taking advantage of the possibilities of hyperthermia. 


\section{Acknowledgements}

This work was supported by FEDER through the COMPETE/QREN/EU Program and by the Portuguese Foundation for Science and Technology (FCT) in the framework of the Strategic Project of CFUM [PEst-C/FIS/UI0607/2013 (F-COMP-01-0124-FEDER022711)] and through the research project PTDC/QUI/81238/2006 (FCOMP-01-0124FEDER-007467). FCT, POPH-QREN and FSE are acknowledged for the PhD grant of A.R.O. Rodrigues (SFRH/BD/90949/2012) and for financial support to MAP-Fis PhD Programme.

\section{References}

[1] B. P. Timko, K. Whitehead, W. Gao, D. S. Kohane, O. Farokhzad, D. Anderson, R. Langer, Annu. Rev. Mater. Res. 41 (2011) 1-20.

[2] S. Mornet, S. Vasseur, F. Grasset, E. Duguet, J. Mater. Chem. 14 (2004) 2161-2175.

[3] M. Mezei, V. Gulasekharam, J. Pharm. Pharmacol. 34 (1982) 473-474.

[4] R. L. Juliano, Trends Pharmacol. Sci. 2 (1981) 39-42.

[5] G. Poste, C. Cucana, A. Raz, P. Bugelski, R. Kirsh, I.J. Fidler, Cancer Res. 24 (1982) 14121422.

[6] T. L. Andresen, S. S. Jensen, K. Jorgensen, Prog. Lipid Res. 44 (2005) 68-97.

[7] N. A. Ochekpe, P. O. Olorunfemi, N. C. Ngwuluka, Trop. J. Pharm. Res. 8 (2009) 265-274.

[8] A. S. Lubbe, C. Bergemann, J. Brock, D. G. McClure, J. Magn. Magn. Mater. 194 (1999) 149-155.

[9] S. Dandamudi, R. B. Campbell, Biomaterials 28 (2007) 4673-4683.

[10] H.-J. Weinmann, W. Ebert, B. Misselwitz, H. S.-Willich, Eur. J. Radiol. 46 (2003) 33-44.

[11] N. Nuytten, M. Hakimhashemi, T. Ysenbaert, L. Defour, J. Trekker, S. J. Soenen, P. Van der Meeren, M. Cuyper, Colloids Surf. B. Biointerfaces 80 (2010) 227-231.

[12] U. I. Tromsdorf, N. C. Bigall, M. G. Kaul, O. T. Bruns, M. S. Nikolic, B. Mollwitz, R. A. Sperling, R. Reimer, H. Hohenberg, W. J. Parak, S. Forster, U. Beisiegel, G. Adam, H. Weller, Nano Lett. 7 (2007) 2422-2427.

[13] E. Amstad, J. Kohlbrecher, E. Müller, T. Schweizer, M. Textor, E. Reimhult, Nano Lett. 11 (2011) 1664-1670.

[14] G. Gregoriadis, Trends Biotechnol. 13 (1995) 527-537.

[15] S. Dandamudi, R. B. Campbell, Biochim. Biophys. Acta 1768 (2007) 427-438. 
[16] D. L. Huber, Small 1 (2005) 482-501.

[17] K. M. Krishnan, IEEE Trans. Magn. 46 (2010) 2523-2559.

[18] P. Tartaj, M. P. Morales, S. V.-Verdaguer, T. G.-Carreno, C. J. Serna, J. Phys. D: Appl. Phys. 36 (2003) 182-197.

[19] M. Faraji, Y. Yamini, M. Rezaee, J. Iran Chem. Soc. 7 (2010) 1-37.

[20] C. M. Niemeyer, Angew. Chem. Int. Ed. 40 (2001) 4128-4158.

[21] I. M. Hafez, S. Ansell, P. R. Cullis, Biophys. J. 79 (2000) 1438-1446.

[22] J. B. Sun, J. H. Duan, S. L. Dai, J. Ren, L. Guo, W. Jiang, Y. Li, Biotechnol. Bioeng. 101 (2008) 1313-1320.

[23] Y. Malam, M. Loizidou, A. M. Seifalian, Trends Pharmacol. Sci. 30 (2009) 592-599.

[24] N. Sounderya, Y. Zhang, Recent Patents Biomed. Eng. 1 (2008) 34-42.

[25] C. Sun, J.S.H. Lee, M. Zhang, Adv. Drug Deliv. Rev. 60 (2008) 1252-1265.

[26] D. M. Huang, T. H. Chung, Y. Hung, F. Lu, S.-H. Wu, C. Y. Mou, M. Yao, Y. C. Chen, Toxicol. Appl. Pharmacol. 231 (2008) 208-215.

[27] T. Kim, E. Momin, J. Choi, T. Kim, E. Momin, J. Choi, K. Yuan, H. Zaidi, J. Kim, M. Park, N. Lee, M. T. McMahon, A. Quinones-Hinojosa, J. W. M. Bulte, T. Hyeon, A. A. Gilad, J. Am. Chem. Soc. 133 (2011) 2955-2961.

[28] A. Akbarzadeh, M. Samiei, S. Davaran, Nanoscale Res. Lett. 7 (2012) 144-176.

[29] R. Kotitz, W. Weitschies, L. Trahms, W. Brewer, W. Semmler, J. Magn. Magn. Mater. 194 (1999) 62-68.

[30] T. T. Y. Tan, S. Liu, Y. Zhang, M. Y. Han, S. T. Selvan in Comprehensive Nanoscience and Technology, D. Andrews, G. Scholes and G. Wiederrecht, Eds., Academic Press, 2010.

[31] D.-H. Chen, C.-H. Hsieh, J. Mater. Chem. 12 (2002) 2412-2415.

[32] S. Jain, V. Mishra, P. Singh, P. K. Dubey, D. K. Saraf, S.P. Vyas, Int. J. Pharm. 261 (2003) 43-55.

[33] C. J. Meledandri, T. Ninjbadgar, D. F. Brougham, J. Mater. Chem. 21 (2011) 214-222.

[34] S. Santra, R. Tapec, N. Theodoropoulou, J. Dobson, A. Hebard, W. Tan, Langmuir 17 (2001) 2900-2906.

[35] S. Batzri, E. D. Korn, Biochim. Biophys. Acta 298 (1973) 1015-1019.

[36] J. M. H. Kremer, M. W. J. vd Esker, C. Pathmamanoharan, P. H. Wiersema, Biochemistry 16 (1977) 3932-3935.

[37] Y. Tamba, H. Terashima, M. Yamazaki, Chem. Phys. Lipids 164 (2011) 351-358.

[38] T. Tanaka, Y. Tamba, S. Md. Masum, Y. Yamashita, M. Yamazaki, Biochim. Biophys. Acta 1564 (2002) 173-182. 
[39] B. Valeur, Molecular Fluorescence - Principles and Applications, Wiley-VCH, Weinheim, 2002.

[40] J. N. Demas, G. A. Crosby, J. Phys. Chem. 75 (1971) 991-1024.

[41] S. Fery-Forgues, D. Lavabre, J. Chem. Educ. 76 (1999) 1260-1264.

[42] I. Johnson, M. T. Z. Spence, Molecular Probes Handbook: A Guide to Fluorescent Probes and Labeling Technologies, 11th Ed., Invitrogen, 2011.

[43] B. Chu, Laser Light Scattering - Basic Principles and Practice, $2^{\text {nd }}$ Edition, Academic Press, New York, 1991.

[44] C. S. Johnson, Jr., D. A. Gabriel, Laser Light Scattering, Dover, New York, 1994.

[45] P. J. Patty, B. J. Frisken, Appl. Opt. 45 (2006) 2209-2216.

[46] C. F. Bohren, D. R. Huffman, Absorption and Scattering of Light by Small Particles, Wiley-VCH, New York, 2007.

[47] S. Park, Y. K. Lee, M. Jung, K. H. Kim, N. Chung, E. K. Ahn, Y. Lim, K. H. Lee, Inhal. Toxicol. 19 (2007) Suppl. 1, 59-65.

[48] M. Ahamed, Toxicol. in Vitro 25 (2011) 930-936.

[49] P. N. Prasad, Nanophotonics, Wiley-Interscience, New Jersey, 2004.

[50] Y. Kobayashi, M. A. Correa-Duarte, L. M. Liz-Marzán, Langmuir 17 (2001) 6375-6379.

[51] S. Chakrabarty, K. Chatterjee, ISRN Nanotechnol. 2011, Article ID 719027 (2011).

[52] A. L. Aden, M. Kerker, J. Appl. Phys. 22 (1951) 1242-1246.

[53] A. Quirantes Sierra, A. V. Delgado, J. Phys. D: Appl. Phys. 30 (1997) 2123-2131; http://www.ugr.es/ aquiran/ciencia/codigos/bart.f

[54] E. D. Palik, Handbook of Optical Constants of Solids, Elsevier Science \& Tech., 1985.

[55] I. H. Malitson, J. Opt. Soc. Am. 55 (1965) 1205-1208.

[56] M. Ardhammar, P. Lincoln, B. Nordén, PNAS 99 (2002) 15313-15317.

[57] M. Benelmekki, A. Montras, A.J. Martins, P.J.G. Coutinho, Ll. M. Martinez, J. Magn. Magn. Mater. 323 (2011) 1945-1949.

[58] J. P. Chen, C. M. Sorensen, K. J. Klabunde, G. C. Hadjipanayis, E. Devlin, A. Kostikas, Phys. Rev. B 54 (1996) 9288-9296.

[59] H. Curtis, N. Barnes, Biology, $5^{\text {th }}$ Edition, Worth Publishers, New York, 1989.

[60] E. M. S. Castanheira, M. S. D. Carvalho, A. R. O. Rodrigues, R. C. Calhelha, M.-J. R. P. Queiroz, Nanoscale Res. Lett. 6 (2011) article 379.

[61] G. Hungerford, E. M. S. Castanheira, M. E. C. D. Real Oliveira, M. G. Miguel, H. D. Burrows, J. Phys. Chem. B 106 (2002) 4061-4069. 
[62] P. Greenspan, S.D. Fowler, J. Lipid Res. 26 (1985) 781-789.

[63] I. G. Krishnamoorthy, J. Phys. Chem. B 105 (2001) 1484-1488.

[64] P. J. G. Coutinho, E. M. S. Castanheira, M. C. Rei, M. E. C. D. Real Oliveira, J. Phys. Chem. B 106 (2002) 12841-12486.

[65] E. Feitosa, F. R. Alves, A. Niemiec, M. E. C. D. Real Oliveira, E. M. S. Castanheira, A. L. F. Baptista, Langmuir 22 (2006) 3579-3585.

[66] D. K. Struck, D. Hoekstra, R. E. Pagano, Biochemistry 20 (1981) 4093-4099. 